\title{
Prevalence of Scabies and Its Associated Factors Among School-Age Children in Arba Minch Zuria District, Southern Ethiopia, 2018.
}

\section{Eyayou Girma ( $\nabla$ samigirma02@gmail.com )}

Arba Minch University https://orcid.org/0000-0003-4878-5778

Chuchu Churko

Arba Minch University

Amsalu Alagaw

Arba Minch University

Desta Haftu

Arba Minch University

Abayneh Tunje

Arba Minch University

Behailu Tsegaye

Arba Minch University

\section{Research}

Keywords: scabies, school-age children, Arba Minch Zuria woreda.

Posted Date: October 13th, 2020

DOI: https://doi.org/10.21203/rs.3.rs-88911/v1

License: @ (i) This work is licensed under a Creative Commons Attribution 4.0 International License. Read Full License 


\section{Abstract}

Background: Scabies, a common human skin disease with a prevalence range of $0.2 \%$ to $71.4 \%$ in the world. It can have a considerable impact on general health leading to illness and death not only through the direct effect of its infestation and as a result of secondary bacterial infection. This study aimed to assess the prevalence of scabies and its associated factors among school-age children in Arba Minch Zuria district, Gamo zone, Southern Ethiopia.

Methods: A community-based cross-sectional study was carried out in 825 school-age children from February 20 to March 30, 2018. A multi-stage sampling technique was used to select study populations. Logistic regression analysis was used to identify factors associated with scabies. Findings were presented using $95 \% \mathrm{Cl}$ of Crude Odds Ratios (COR) and Adjusted Odds Ratios (AOR). To declare statistical significance, a p-value of less than 0.05 was used.

Result: A total of 825 children participated in the study with a response rate of $97.6 \%$. The overall prevalence of scabies was $16.4 \%$ [95\% Cl: 13.9\%, 18.9\%]. Overcrowding index, family history of itching in the past two weeks, wealth index, knowledge of scabies, climatic zone (living in the highland area), frequency of washing body, frequency of washing clothes, fingernails cutting practice, history of skin contact with scabies patient, washing hair more than once weekly, and sharing of clothes were significantly associated scabies disease.

Conclusion: In conclusion, the prevalence of scabies in Arba Minch lies at $16.4 \%$ in the global scabies range from $0.2 \%$ to $71.4 \%$. The prevalence was highest in highlands followed by midland and then lowland. This represents a significant scabies burden which we recommend warrants health service intervention.

\section{Background}

The skin is the largest body organ and is a sensitive indicator of a child's general health. Skin disorders are the most common health problems among children. Skin disorder may cause emotional and psychological stress for the child and family [1]. Scabies is a contagious skin infestation caused by the parasite Sarcoptes scabiei. It is the most common ectoparasitic dermatosis seen in clinical practices [2].

The International Alliance for the Control of Scabies, a newly formed organization, proposes to achieve scabies control in vulnerable communities in 2013 [3]. Recently in the tenth strategic and technical advisory group for neglected tropical diseases World Health Organization (WHO) added scabies to the list of "Neglected Tropical Diseases", thereby recognizing its impact on human health [4].

Ethiopia has been affected by natural disasters such as the El Niño weather phenomenon, leading to severe drought and scabies outbreaks [5]. The country faced a scabies outbreak in many parts of the region due to this weather event. [5]. Ethiopia has developed a guideline to assist with control and to attempt to prevent scabies outbreaks in response to these outbreaks. The recommended interventions include Health, Water, Sanitation, and Hygiene (WASH), and communication for development. Considering scabies as the water washed disease, the key intervention is the provision of access to sufficient safe water for personal hygiene: washing of clothing, washing of body using soaps especially the affected areas, appropriate hand washing at critical times, clothing or bedding that were used by an infected individual during and before effective treatment should be dried for 3 days in the sun to allow time for mites and eggs to die. The communication strategies for the prevention of scabies need integrated and multi-level interventions comprising advocacy, social mobilization, social and behavioral change communication at different levels and capacities should be provided in Ethiopia [6].

Scabies is a common problem in school-age children and there is a limited study done on scabies in the study area. Therefore, this study aimed to assess the prevalence of scabies and its associated factors among school-age children 
in Arba Minch zuria district, Gamo zone Southern Ethiopia.

\section{Methods}

\section{Study area and period}

A community-based cross-sectional study was conducted from March 1-30, 2018 in Arba Minch Zuria district, Gamo Gofa Zone. The district is located 505 kilometers south of Addis Ababa, the capital city of Ethiopia and the district has 29 kebeles. According to the central statistical agency of Ethiopia in 2007 the total population of the district was 165,680 of which 82,751 were males and 82,929 were females. The district has 7 health centers and 40 health posts.

\section{Population}

The source populations were all children aged 5-14 years in the selected rural kebeles who fulfill selection criteria during the study period. All children aged 5 to 14 years in the selected kebeles were included in the study, and children who were severely ill excluded from the study.

\section{Sample size and sampling}

The sample size was determined using single population proportion formula with the assumption of $95 \%$ confidence interval $(\mathrm{Cl})$, by taking prevalence of scabies $50 \%$, design effect $[\mathrm{DE}]=2$, a none response rate of $10 \%$. The final, the calculated sample size was 845 . The study participants were recruited using a multi-stage sampling technique. First based on their climatic zone kebeles were stratified into three. Then kebeles were selected randomly by using the lottery method from each stratum. Sampling frame obtained from the woreda education office for each kebele. Then the sample was allocated by proportionate allocation for all selected kebeles.

By using simple random sampling the first household was chosen by the direction of the pen point as a starting point. If in case more than one eligible respondent was found in the household, only one respondent was chosen by the lottery method.

\section{Data collection}

Socio-demographic characteristics and associated risk factors were collected using a structured questionnaire by trained health care providers who were BSc degree holders. A structured questionnaire was used to interview parents and children in their homes. Physical examination was undertaken on respondents who had an itchy vesicular skin rash, burrows, papules, and nodules by trained BSc nurses. The diagnosis was ascertained, based on the Mali clinical algorithm [7]. A skin scraping was not feasible in this setting.

\section{Operational definitions}

Scabies: in this study scabies is defined as the presence of persistent pruritic rash with itching increasing at night which are notified at least at two specific body sites (on the wrist, sides and web spaces of the fingers, the axillae, periareolar, per umbilical, genitalia area, abdomen, and buttock areas) with or without a history of pruritus in the close entourage [7].

Good knowledge: Those mother/caregiver who answered above the mean of the knowledge questions.

Poor knowledge: Those mother/caregiver who answered below the mean of the knowledge questions.

School-age children: children who were in the age group 5-14 years old [8]. 
Infrequent bathing - showering frequency less than once per week in the past month [9].

Infrequent washing clothes - washing clothes less than once per week in the past month [9].

Infrequent changing clothes - changing clothes less than once per week in the past month [9].

Overcrowding index: was calculated by dividing the number of usual residents in a house by the number of bedrooms in the house. If it is more than 1.5 overcrowded and if it is less than or equal to 1.5 not overcrowded [10].

\section{Data quality control}

To ensure the quality of data, a questionnaire was prepared in the English language, translated to Amharic, and retranslated back to English by another person who can speak both languages. To make sure that the questionnaire is appropriate and understandable; it was pre-tested on $5 \%$ of the sample size. The training was given for supervisors and data collectors for one day. Regular supervision was carried out during the data collection period'. 'Collected data were checked for completeness and consistency daily.

\section{Data analysis}

Epi info version 7 was used for entering, coding, cleaning the collected data that were analyzed using SPSS version 20. In the univariate analysis, descriptive statistics were conducted to explore frequency distribution, central tendency, variability (dispersion), and overall distribution of independent variables. Bivariate analysis was done to determine the associations between each independent variable and outcome variable. All associated factors with a p-value of less than 0.25 during bivariate analysis and biologically plausible factors were entered into a multivariable logistic regression model. Odds ratio with $95 \%$ confidence intervals was used to see the strength of the association between different variables. P-value and $95 \%$ confidence interval $(\mathrm{Cl})$ for odds ratio $(\mathrm{OR})$ were used in deciding the significance of the associations. The wealth index was calculated by using the principal component analysis method (PCA) and constructed as lowest, second, middle, fourth, and highest.

\section{Results}

Socio-demographic characteristics of the respondents

A total of 825 (response rate of $97.6 \%$ ) school-age children participated in the study. Of the total children, $53.2 \%$ $(439 / 825)$ were men. Four hundred fifty-three $54.9 \%$ of the children were in the age group between $10-14$ years old. The majority $81.6 \%(673 / 825)$ of the study subjects attended school whereas $18.4 \%(152 / 825)$ did not. The majority $91.3 \%$ of the respondents were Gamo ethnic group, $5.6 \%$ were Wolayta, $1.8 \%$ Amhara and the rest $1.3 \%$ others. More than half (60.1\%) was a follower of Protestant Christianity followed by Orthodox Christianity (38.8\%) and 1.1\% others. Regarding family education, $50.0 \%$ of the mothers had no formal education, $44.2 \%$ had primary education, and $5.8 \%$ had secondary and higher educational levels. $43.9 \%$ of children's father had no formal education, $41.1 \%$ had attended primary education and $15 \%$ had secondary and higher educational status. The majority $92.6 \%(764 / 825)$ of children's mothers interviewed in this study were housewives, $5.8 \%$ were merchants, and $1.6 \%$ employed Gov't/NGO. On the other hand, $91.6 \%(756 / 825)$ of the children's father's farmer, $6.3 \%$ (52/825), and 2.1\% (17/825) were Merchant and employed respectively (Table 1$)$. 
Table 1

Socio-demographic characteristics of the study subjects in Arba Minch zuria district, SNNPR, 2018; N $=825$.

\begin{tabular}{|c|c|c|c|c|}
\hline \multicolumn{3}{|c|}{ Socio-demographic variables Category } & Number & Percent (\%) \\
\hline \multirow{2}{*}{\multicolumn{2}{|c|}{ Sex of the children }} & male & 439 & 53.2 \\
\hline & & female & 386 & 46.8 \\
\hline \multirow{2}{*}{\multicolumn{2}{|c|}{ Age group }} & $5-9$ years & 372 & 45.1 \\
\hline & & $10-14$ years & 453 & 54.9 \\
\hline \multirow{2}{*}{\multicolumn{2}{|c|}{ Children attending school }} & Yes & 673 & 81.6 \\
\hline & & No & 152 & 18.4 \\
\hline \multirow{4}{*}{\multicolumn{2}{|c|}{ Ethnicity }} & Gamo & 753 & 91.3 \\
\hline & & Wolayta & 46 & 5.6 \\
\hline & & Amhara & 15 & 1.8 \\
\hline & & Others* & 11 & 1.3 \\
\hline \multirow{3}{*}{\multicolumn{2}{|c|}{ Religion }} & Protestant & 496 & 60.1 \\
\hline & & Orthodox & 320 & 38.8 \\
\hline & & Others ${ }^{\star \star}$ & 9 & 1.1 \\
\hline \multirow[t]{6}{*}{ Family educations } & \multirow[t]{3}{*}{ Childs mother } & No formal education & 412 & 50.0 \\
\hline & & Primary education & 365 & 44.2 \\
\hline & & Secondary and above & 48 & 5.8 \\
\hline & \multirow[t]{3}{*}{ Childs father } & No formal education & 362 & 43.9 \\
\hline & & Primary education & 339 & 41.1 \\
\hline & & Secondary and above & 124 & 15 \\
\hline \multirow[t]{6}{*}{ Occupational status of family } & \multirow[t]{3}{*}{ Childs mother } & Housewife & 764 & 92.6 \\
\hline & & Merchant & 48 & 5.8 \\
\hline & & Employed Govt/NGO & 13 & 1.6 \\
\hline & \multirow[t]{3}{*}{ Childs father } & Farmer & 756 & 91.6 \\
\hline & & Merchant & 52 & 6.3 \\
\hline & & Employed Govt/NGO & 17 & 2.1 \\
\hline \multirow{3}{*}{\multicolumn{2}{|c|}{ Climatic zone }} & Lowland & 325 & 39.4 \\
\hline & & Mid land & 416 & 50.4 \\
\hline & & High land & 84 & 10.2 \\
\hline \multicolumn{2}{|l|}{ Wealth Index } & 1 & 156 & 18.9 \\
\hline
\end{tabular}




\begin{tabular}{|llll|}
\hline Socio-demographic variables Category & & Number & Percent (\%) \\
\hline & 2 & 178 & 21.5 \\
\hline *zeyse, Gidicho, Gofa **muslim, Catholic, Hawariyat & 3 & 158 & 19.2 \\
\hline
\end{tabular}

Prevalence of scabies among school-age children

The overall prevalence of scabies among school-age children 16.4\% (135/825) [95\% Cl: 13.9\%, 18.9\%] scabies cases were identified. Out of which 45 (13.8\%) [95\% Cl: 10.05\%, 17.55\%] were found in lowland, $65(15.6 \%)[12.11 \%, 19.09 \%]$ in midland and 25 (29.8\%) [95\% Cl: 20.019\%, 39.58\%] found in highland area. The prevalence of scabies among females was higher than male children, $17.1 \%$ and $15.7 \%$ respectively.

Home environment-related factors

More than three-quarters of the sample, 638/825 (77.3\%) had a family size of greater than or equal to five, and only $187 / 825(22.7 \%)$ had less than five family members with the mean family size of 6 and standard deviation 1.95. 80.2\% $(662 / 825)$ of the children share a bed with others whereas $19.8 \%(163 / 825)$ did not share their bed with other family members. From those families who had animals in their home, 366/825 (55.5\%) of the children look animals and 294/825 (44.5\%) did not deal with animals in their homes. 474/825 (57.5\%), 239/825 (28.9\%), and 112/825 (13.6\%) of the respondents use electricity, kerosene light, and solar light in their home respectively. Majority 561 (68\%) of the respondent's house was covered by an iron sheet and 264 (32\%) did not. Almost all 815 (98.8\%) of the family was living in a house built by soft bricks and $10(1.2 \%)$ living in a hard brick.

The majority of $586 / 825$ (71\%) of the respondents use river/pond as a source of water for personal hygiene, 136/825 (16.5\%) use pipe/tap water and the rest 103/825 (12.5\%) use well/spring water in the study area. Regarding knowledge $636 / 825(77.1 \%)$ of the family had good knowledge about scabies and 189/825 (22.9\%) had poor knowledge. About $747(90.5 \%)$ had a water source near their home $<30$ minutes and $78(9.5 \%)$ had a water source far away from home (Table 2). 
Table 2

Home environment factors of the respondents in the study area, 2018; $\mathrm{N}=825$.

\begin{tabular}{|c|c|c|c|}
\hline \multicolumn{2}{|l|}{ Variables Category } & \multirow{2}{*}{$\begin{array}{l}\text { Frequency } \\
187\end{array}$} & \multirow{2}{*}{$\begin{array}{l}\text { Percentages } \\
22.7\end{array}$} \\
\hline Family size & $<5$ & & \\
\hline & $>=5$ & 638 & 77.3 \\
\hline \multirow[t]{2}{*}{ Bed sharing } & Yes & 662 & 80.2 \\
\hline & No & 163 & 19.8 \\
\hline \multirow[t]{2}{*}{ Dealing with animals } & Yes & 366 & 55.5 \\
\hline & No & 294 & 44.5 \\
\hline \multirow[t]{3}{*}{ Type of light } & Kerosene & 239 & 28.9 \\
\hline & Solar & 112 & 13.6 \\
\hline & Electricity & 474 & 57.5 \\
\hline \multirow[t]{2}{*}{ House type iron sheet covered } & Yes & 561 & 68 \\
\hline & No & 264 & 32 \\
\hline \multirow[t]{2}{*}{ Type of house built by } & Soft bricks & 815 & 98.8 \\
\hline & Hard bricks & 10 & 1.2 \\
\hline \multirow[t]{3}{*}{ Source of water for personal hygiene } & Pipe/tap water & 136 & 16.5 \\
\hline & Well/spring & 103 & 12.5 \\
\hline & River/pond & 586 & 71 \\
\hline \multirow[t]{2}{*}{ Knowledge status } & Good & 636 & 77.1 \\
\hline & Poor & 189 & 22.9 \\
\hline \multirow[t]{2}{*}{ Distance of water source from home } & Near home $/<=30$ minute & 747 & 90.5 \\
\hline & Far away from home $>30$ minute & 78 & 9.5 \\
\hline
\end{tabular}

Regarding personal hygiene, $85.8 \%(708 / 825)$ of the respondents wash their body frequently and $14.2 \%(117 / 825) \mathrm{had}$ responded that they wash their body infrequently. The majority $80.2 \%(662 / 825)$ of the participants wash their clothes frequently, $19.8 \%$ (163/825) wash their clothes infrequently. More than three quarters $83.5 \%(689 / 825)$ of the respondents changed into clean clothes frequently whereas the rest $16.5 \%(136 / 825)$ changed clothes infrequently. $56.7 \%$ (468/825), 280/825 (34\%), and 77/825 (9.3\%) of the children wash their hair 1-7 days, 7-14 days, and more than 14 days respectively. $627 / 825(76 \%)$ of the children share their clothes with any other person whereas $198 / 825$ (24\%) did not share their clothes. About 563 (68.2\%) of the children cut their fingernails short/trimmed and 262/825 (31.8\%) did not (Table 3). 
Table 3

personal hygiene and sanitation characteristics of the respondents, 2018; $\mathrm{N}=$ 825.

\begin{tabular}{|llll|}
\hline Variables Category & & Frequency & Percentages \\
\hline Body washing & Frequently & 708 & 85.8 \\
\cline { 2 - 4 } Washing clothes & Infrequently & 117 & 14.2 \\
\hline Changing of clothes & Frequently & 662 & 80.2 \\
\cline { 2 - 4 } & Infrequently & 163 & 19.8 \\
\hline Frequency of hair washing & Frequently & 689 & 83.5 \\
\cline { 2 - 4 } & Infrequently & 136 & 16.5 \\
\cline { 2 - 4 } & $1-7$ days & 468 & 56.7 \\
\hline Share clothes with other & $7-14$ days & 280 & 34 \\
\cline { 2 - 4 } & $>14$ days & 77 & 9.3 \\
\hline Fingernails cut short/trimmed & Yes & 198 & 24 \\
\cline { 2 - 4 } & No & 627 & 76 \\
\cline { 2 - 4 } & No & 262 & 31.8 \\
\cline { 2 - 4 } & & & 68.2 \\
\hline
\end{tabular}

Knowledge about scabies among children's family

From the total respondents, $805 / 825$ [97.6\%] knew the signs and symptoms of scabies, and $78.7 \%$ knew parts of the body that are affected by scabies as finger webs, armpits, genitalia, abdomen, breast, waist and knees, 143/825 [17.3\%] knew that scabies affects parts of body that are mostly covered and 33 [4\%] said it affects mostly at genitalia area (Table 4).

Regarding respondent's knowledge about transmission, 545/825 [66.1\%] knew scabies transmitted through skin to skin contact and infected fomites like clothing, bed linen, 213 [25.8\%] through the skin to skin contact only, and 67 [8.1\%] through fomites (Table 4). 
Table 4

Knowledge of respondents about scabies infection in the study area, 2018; N = 825 .

\begin{tabular}{|c|c|c|c|}
\hline Variables & Categories & Frequency & $\begin{array}{l}\% \\
\text { [percentage] }\end{array}$ \\
\hline \multirow[t]{2}{*}{ Ever heard about scabies } & Yes & 788 & 95.5 \\
\hline & No & 37 & 4.5 \\
\hline \multirow[t]{3}{*}{ Etiology of the disease } & Parasite & 23 & $2.8 \%$ \\
\hline & Germs & 246 & 29.8 \\
\hline & The effect of scratching & 556 & $67.4 \%$ \\
\hline \multirow[t]{2}{*}{ Signs and symptoms of scabies } & Itchy skin rash worsens at night & 805 & 97.6 \\
\hline & Don't know & 20 & 2.4 \\
\hline \multirow[t]{3}{*}{$\begin{array}{l}\text { Parts of the body that are affected } \\
\text { by scabies }\end{array}$} & $\begin{array}{l}\text { Finger webs, armpits, genitalia, buttocks, } \\
\text { abdomen, breast, knees }\end{array}$ & 649 & $78.7 \%$ \\
\hline & Parts that are mostly covered & 143 & $17.3 \%$ \\
\hline & Mostly at genitalia & 33 & $4 \%$ \\
\hline \multirow[t]{3}{*}{ Transmission way } & $\begin{array}{l}\text { Skin to skin contact and through contaminated } \\
\text { fomites }\end{array}$ & 545 & $66.1 \%$ \\
\hline & Through skin contact only & 213 & $25.8 \%$ \\
\hline & Through fomites only & 67 & $8.1 \%$ \\
\hline \multirow[t]{3}{*}{ Sufferer from scabies } & All age group but mostly teenagers & 571 & 69.2 \\
\hline & Teenagers only & 126 & 15.3 \\
\hline & Only in a certain age groups & 128 & 15.5 \\
\hline \multirow[t]{2}{*}{ Exchanging clothes spread scabies } & Yes & 604 & 73.2 \\
\hline & No & 221 & 26.8 \\
\hline \multirow{2}{*}{$\begin{array}{l}\text { Scabies be harmful to the health of } \\
\text { the skin }\end{array}$} & Yes & 714 & 86.5 \\
\hline & No & 111 & 13.5 \\
\hline \multirow{3}{*}{$\begin{array}{l}\text { Scabies patient need to be } \\
\text { quarantined }\end{array}$} & No just need regular treatment simultaneous & 570 & 69.1 \\
\hline & Just keep distant from scabies patient & 180 & 21.8 \\
\hline & Need regular treatment only & 75 & 9.1 \\
\hline \multirow{3}{*}{$\begin{array}{l}\text { Ways to break the chain of scabies } \\
\text { transmission }\end{array}$} & Disinfect fomites and give treatment & 454 & $55 \%$ \\
\hline & Keep distant from scabies patient & 125 & $15.2 \%$ \\
\hline & Need regular treatment only & 246 & $29.8 \%$ \\
\hline \multirow{2}{*}{$\begin{array}{l}\text { Drying mattress and pillows } \\
\text { prevent scabies }\end{array}$} & Yes & 462 & $56 \%$ \\
\hline & No & 363 & $44 \%$ \\
\hline Prevention measure for scabies & $\begin{array}{l}\text { Frequent bathing and avoid physical contact } \\
\text { with scabies patient }\end{array}$ & 439 & $53.2 \%$ \\
\hline
\end{tabular}




\begin{tabular}{|lllc|}
\hline Variables & Categories & Frequency & $\begin{array}{l}\% \\
\text { [percentage] }\end{array}$ \\
\hline & $\begin{array}{l}\text { Frequent bathing and cleanliness of clothes } \\
\text { only }\end{array}$ & 239 & $29 \%$ \\
\hline Keep fomites from contamination only & 147 & $17.8 \%$ \\
\hline
\end{tabular}

\section{Factors associated with scabies disease}

Overcrowding index more than 1.5 [AOR $=5.433,95 \% \mathrm{Cl}: 2.264,13.04]$ were 5 times more likely to have scabies than whose overcrowding index less than or equals to 1.5. Those children whose family member or entourage complaining of itchy skin rash in the past two weeks $[\mathrm{AOR}=6.99,95 \% \mathrm{Cl}: 2.81,17.406]$ were 7 times high risk of getting scabies when compared to no complaining in the family about itching. Families who had fourth wealth quintile (AOR $=5.542,95 \% \mathrm{Cl}$ : $1.402,12.91$ ) were 5.5 times more likely to have scabies when compared to the wealth quintile highest. Children whose family had poor knowledge about scabies [AOR $=5.2,95 \% \mathrm{Cl}: 2.188,12.358]$ were 5 more likely to be affected by scabies disease than those whose family had good knowledge. This study also revealed that children who were living in the lowland $[A O R=0.306,95 \% \mathrm{Cl}=0.109,0.588] 69.4 \%$ reduced risk for scabies when compared to highland whereas children who were living in midlands $[\mathrm{AOR}=0.053,95 \% \mathrm{Cl}: 0.012,0.24]$ were $94.7 \%$ less prone to scabies as compared to children living in highlands.

Those children who wash their clothes infrequently were 3.5 times higher risk of getting sick by scabies when compared to those children washing their clothes frequently [AOR $=3.53,95 \% \mathrm{Cl}: 1.454,8.566]$. Children who wash their body infrequently had 6 times more prone to scabies than children who frequently wash their body $[\mathrm{AOR}=6.321,95 \% \mathrm{Cl}$ : $2.312,17.284]$. This study identified that children who share their clothes with others were 6 times more likely to develop scabies than children who did not share clothes [AOR $=6.013,95 \% \mathrm{Cl}: 2.51,14.4]$.

This study identified that children who had a history of contact were 10 times more prone to scabies than those who did not have contact history $(A O R=9.579,95 \% \mathrm{Cl}: 4.03,17.22)$. Those children who wash their hair $7-14$ days and $>14$ days $(\mathrm{AOR}=7.118,95 \% \mathrm{Cl}: 2.63,19.268)(\mathrm{AOR}=5.11,95 \% \mathrm{Cl}: 1.38,18.899)$ were 7 and 5 times more likely to be affected than those children who wash hair 1-7 days respectively. Children who did not cut their finger short/trimmed were 7.6 times more prone to scabies when compared to children who cut short/trimmed (AOR $=7.6,95 \% \mathrm{Cl}: 3.169,18.245)$ (Table 5). 
Table 5

Multivariable logistic regression analysis of factors associated with scabies among school-age children in Arba Minch zuria district, 2018; $\mathrm{N}=825$.

\begin{tabular}{|c|c|c|c|c|c|c|}
\hline \multirow[t]{2}{*}{ Variables } & \multirow[t]{2}{*}{ Categories } & \multicolumn{3}{|l|}{ Scabies } & \multirow[t]{2}{*}{ COR $[95 \% \mathrm{Cl}]$} & \multirow[t]{2}{*}{ AOR [95\%Cl] } \\
\hline & & Yes & No & Total & & \\
\hline \multirow{2}{*}{$\begin{array}{l}\text { Overcrowding } \\
\text { Index }\end{array}$} & $<=1.5$ & $42[8.1 \%]$ & $476[91.9 \%]$ & 518 & 1 & \\
\hline & $>1.5$ & $93[30.3 \%]$ & $214[69.7 \%]$ & 307 & $\begin{array}{l}4.925[3.306 \\
7.336]\end{array}$ & $5.433[2.264,13.04]^{\star \star \star}$ \\
\hline \multirow{2}{*}{$\begin{array}{l}\text { Fingernails cut } \\
\text { short/trimmed }\end{array}$} & Yes & $24[4.3 \%]$ & $539[95.7 \%]$ & 563 & 1 & \\
\hline & No & $111[42.4 \%]$ & $151[57.6 \%]$ & 262 & $\begin{array}{l}16.509[10.249 \\
26.592]\end{array}$ & $7.6[3.169,18.245]^{*}$ \\
\hline \multirow{2}{*}{$\begin{array}{l}\text { Family } \\
\text { member/entourage } \\
\text { complaining of } \\
\text { itching in the past } \\
\text { two weeks }\end{array}$} & Yes & $83[45.4 \%]$ & $100[54.6 \%]$ & 183 & $\begin{array}{l}9.417[6.273 \\
14.138]\end{array}$ & $6.99[2.81,17.406]^{\star \star \star}$ \\
\hline & No & $52[8.1 \%]$ & $590[91.9 \%]$ & 642 & 1 & \\
\hline \multirow[t]{2}{*}{ Knowledge status } & $\begin{array}{l}\text { Good } \\
\text { knowledge }\end{array}$ & $48[17.5 \%]$ & $588[92.5 \%]$ & 636 & 1 & \\
\hline & $\begin{array}{l}\text { Poor } \\
\text { knowledge }\end{array}$ & $87[46 \%]$ & $102[54 \%]$ & 189 & $\begin{array}{l}10.449[6.932 \\
15.749]\end{array}$ & $5.20[2.188,12.358]^{\star \star \star}$ \\
\hline \multirow[t]{2}{*}{$\begin{array}{l}\text { Have you infected } \\
\text { by scabies before? }\end{array}$} & Yes & $24[50 \%]$ & $24[50 \%]$ & 48 & $\begin{array}{l}6[3.292 \\
10.937]\end{array}$ & $0.921[0.17,2.314]$ \\
\hline & No & $111[14.3 \%]$ & $666[85.7 \%]$ & 777 & 1 & \\
\hline \multirow[t]{3}{*}{ Climatic zone } & Lowland & $45[13.8 \%]$ & $280[86.2 \%]$ & 325 & $\begin{array}{l}0.379[0.216 \\
0.667]\end{array}$ & $0.306[0.109,0.588]^{\star \star}$ \\
\hline & Midland & $65[15.6 \%]$ & $351[84.4 \%]$ & 416 & $\begin{array}{l}0.437[0.255 \\
0.748]\end{array}$ & $0.053[0.012,0.24]^{\star \star \star}$ \\
\hline & Highland & $25[29.8 \%]$ & $59[70.2 \%]$ & 84 & 1 & 1 \\
\hline \multirow[t]{2}{*}{$\begin{array}{l}\text { Sharing of bed } \\
\text { with others }\end{array}$} & Yes & $122[18.5 \%]$ & $540[81.6 \%]$ & 662 & $\begin{array}{l}2.607[1.431 \\
4.749]\end{array}$ & $0.954[0.251,3.635]$ \\
\hline & No & $13[7.9 \%]$ & $150[92 \%]$ & 163 & 1 & 1 \\
\hline \multirow{2}{*}{$\begin{array}{l}\text { How often did the } \\
\text { child change clean } \\
\text { clothes }\end{array}$} & Frequently & 77[11.5\%] & $590[88.5 \%]$ & 712 & 1 & 1 \\
\hline & Infrequently & $58[36.7 \%]$ & $100[63.3 \%]$ & 113 & $\begin{array}{l}4.444[2.975 \\
6.639]\end{array}$ & $0.567[0.174,1.849]$ \\
\hline \multirow{2}{*}{$\begin{array}{l}\text { How often did you } \\
\text { wash your clothes }\end{array}$} & Frequently & $43[6.5 \%]$ & $619[93.5]$ & 662 & 1 & \\
\hline & Infrequently & $92[56.4 \%]$ & $71[43.6 \%]$ & 163 & $\begin{array}{l}\text { 18.653[12.043, } \\
28.890]\end{array}$ & $3.53[1.454,8.566]^{\star}$ \\
\hline \multirow{2}{*}{$\begin{array}{l}\text { How often did you } \\
\text { wash your body }\end{array}$} & Frequently & $61[8.6 \%]$ & $647[91.4 \%]$ & 708 & 1 & \\
\hline & Infrequently & $74[63.2 \%]$ & $43[36.8 \%]$ & 117 & $\begin{array}{l}18.253[11.541 \\
28.869]\end{array}$ & $6.321[2.312,17.284] \star \star$ \\
\hline
\end{tabular}

$P<0.05^{*} p<0.01^{\star *} p<0.001^{*}$; COR means crude odds ratios to see association between the explanatory variable with the outcome variable; AOR means adjusted odds ration which means looking association of the variable with the outcome variable after adjusting other confounding variables. 


\begin{tabular}{|c|c|c|c|c|c|c|}
\hline \multirow[t]{2}{*}{ Sharing of clothes } & No & $33[5.1 \%]$ & $608[94.9 \%]$ & 641 & 1 & 1 \\
\hline & Yes & $102[55.4 \%]$ & $82[44.6 \%]$ & 184 & $\begin{array}{l}22.918[14.537, \\
36.131]\end{array}$ & $6.013[2.51,14.4]^{\star \star \star}$ \\
\hline \multirow{2}{*}{$\begin{array}{l}\text { Distance to fetch } \\
\text { water }\end{array}$} & Near home & $100[13.4 \%]$ & $647[86.6 \%]$ & 747 & 1 & 1 \\
\hline & Far away & $35[44.9 \%]$ & $43[55.1 \%]$ & 78 & $\begin{array}{l}5.266[3.215 \\
8.626]\end{array}$ & $3.106[0.674,14.313]$ \\
\hline \multirow{2}{*}{$\begin{array}{l}\text { History of contact } \\
\text { with scabies } \\
\text { patient }\end{array}$} & No & $18[3.1 \%]$ & $561[96.9 \%]$ & 579 & 1 & 1 \\
\hline & Yes & $117[47.6 \%]$ & $129[52.4 \%]$ & 246 & $\begin{array}{l}28.267[13.307 \\
48.114]\end{array}$ & $9.579[4.03,17.22]^{\star \star \star}$ \\
\hline \multirow{3}{*}{$\begin{array}{l}\text { How often did you } \\
\text { wash hair }\end{array}$} & $1-7$ day & $17[3.6 \%]$ & $451[65.4 \%]$ & 468 & 1 & 1 \\
\hline & 7-14 day & $81[28.9 \%]$ & $199[71.1 \%]$ & 280 & $\begin{array}{l}10.798[6.237 \\
18.694]\end{array}$ & $7.118[2.63,19.268]^{\star \star}$ \\
\hline & $>14$ day & $37[48.1 \%]$ & $40[51.9 \%]$ & 77 & $\begin{array}{l}24.54[12.695 \\
47.434]\end{array}$ & $5.11[1.38,18.899]^{\star \star}$ \\
\hline \multirow[t]{5}{*}{ Wealth Index } & 1 & $27[17.3 \%]$ & $129[82.7 \%]$ & 156 & $\begin{array}{l}1.408[0.765 \\
2.592]\end{array}$ & $0.571[0.146,2.237]$ \\
\hline & 2 & $23[12.9 \%]$ & $155[87.1 \%]$ & 178 & $\begin{array}{l}0.998[0.534 \\
1.868]\end{array}$ & $2.01[0.457,8.87]$ \\
\hline & 3 & $25[15.8 \%]$ & $133[84.2 \%]$ & 158 & $\begin{array}{l}1.265[0.681 \\
2.348]\end{array}$ & $1.48[0.36,6.072]$ \\
\hline & 4 & $38[23.3 \%]$ & $125[76.7 \%]$ & 163 & $\begin{array}{l}2.045[1.149 \\
3.64]\end{array}$ & $5.542[1.402,12.9]^{\star}$ \\
\hline & 5 & $22[12.9 \%]$ & $148[87.1 \%]$ & 170 & 1 & 1 \\
\hline $\begin{array}{l}P<0.05^{*} p<0.01^{\star *} \\
\text { with the outcome } \\
\text { the outcome variab }\end{array}$ & $\begin{array}{l}0.001 * \text {; COF } \\
\text { ble; AOR m€ } \\
\text { fter adjustir }\end{array}$ & $\begin{array}{l}\text { eans crude } \\
\text { s adjusted o } \\
\text { ther confou }\end{array}$ & $\begin{array}{l}\text { Is ratios to } \\
\text { s ration whi }\end{array}$ & $\begin{array}{l}\text { assc } \\
\text { mea }\end{array}$ & $\begin{array}{l}\text { tion between the } \\
\text { ooking associatic }\end{array}$ & $\begin{array}{l}\text { explanatory variable } \\
n \text { of the variable with }\end{array}$ \\
\hline
\end{tabular}

\section{Discussion}

This study was held to assess the prevalence of scabies and to determine possible risk factors among school-age children. Scabies affects children's life such as reducing school attendance due to severe itching. Secondary bacterial complications were another problem that affects scabies patients. This study showed that the overall prevalence of scabies was $16.4 \%$. It is comparable with a study conducted in India wardha district $18 \%$ and with a report from Cameroon 17.8\% [11]. However, it is much lower than the study done at West Bengal India, and Pakistan with a prevalence of $42 \%$ and $47.6 \%$ respectively $(12,13)$. This study also revealed that the prevalence is lower from the study done in Vanuatu, Solomon Island, Sierra Leon displacement camp, and Malaysian welfare home with a prevalence of $24 \%, 25 \%, 86 \%$, and $31 \%$ respectively $[9,12-14]$. This difference might be due to the difference in socio-economic characteristics between the study areas. The climatic condition was significantly associated with scabies infection. This finding was consistent with the study done in Iran and by Hosseini-Shokouh et al $[15,16]$. This might be due to weather change or reduced atmospheric temperature (increased humidity) in the highland area lead to reduce the frequency of bathing of their clothes as well as their body; whereas in the lowland area, people frequently washes their body and clothes due to hot weather condition. This allows the mites do not transmit to others. 
Evidence suggests that overcrowding was one of the main risk factors for scabies infection in different parts of the world including Ethiopia [17-21]. This study also revealed that the overcrowding index was positively associated with scabies. This might be due to frequent body contact with scabies patients and the sharing of clothes in the family.

A study conducted in Cameroon boarding school reported that scabies had no statistically significant association with fingernails cut short [22]. In contrast, this study revealed that children who did not cut their fingernails short/trimmed were significantly related to scabies infestation. This might be explained by fingernails that can hold scabies parasites and transmit scabies disease.

This study showed that children with other family members reporting itchy skin are more prone to scabies infection as the family members highly likely to have scabies.

This finding similar to a study done in Egypt [10], and Tigray regional and SNNPR Ethiopia [23, 24]. This might be due to frequent and prolonged body contact between family members sufficient to pass the mites into other skin.

The present study revealed that children's families who had poor knowledge about scabies were at higher risk of acquiring scabies than those families who had good knowledge. This was in line with a study done in Cempaka District Banjarbaru South Kalimantan [25], and in Ethiopia [17]. This might be because families who knew about the disease take care of the children and themselves from the disease as well as be treated immediately.

Regarding personal hygiene, findings from this study revealed that washing hair more than seven days, infrequent washing of clothes, and infrequent washing of body was positively associated with scabies. It was true for a study conducted in Pakistan, Brazil, Egypt, Amhara, and Tigray region in Ethiopia; they reported that there was a significant association between the factors and scabies [17, 23, 26-29]. The reason might be the respondents had less awareness about the importance of personal hygiene and poor personal hygiene might be a risk factor for the spread of scabies mites.

This study also revealed that children who shared their clothes were statistically significantly associated with a scabies infestation. This finding was consistent with the study done in Doga-Tembi district Tigray [23], Gojjam Amhara region [17], and Egypt [10]. This might due to scabies mites can stay out of human skin for up to 48 hours, physical transmission of the female mites through fomites like clothes possible. Children who had a history of contact with scabies patients in the past two months were 10 times more likely higher when compared to no history of contact. This might be explained by scabies was one of communicable disease which can be transmitted through physical body contact from the infected person to other healthy person. Regarding wealth index, those children families who were the highest wealth quintile had a reduced risk of scabies disease. This might be due to those family who are the highest wealth quintile had good personal hygiene practices like wearing clean clothes, not sharing clothes.

This study further revealed that all children diagnosed scabies had itchy skin rash worsen at night. Body parts affected by scabies were finger webs and ulnar area, auxiliary area, genitalia, on the abdomen, on the shoulder blades, on the elbow, on the buttocks, and the lower legs. In this study, the seasonal variation of the diseases and the confirmatory diagnosis was not addressed.

\section{Conclusion}

In this study, the prevalence of scabies was high among school-age children. Most of the children diagnosed as scabies were living in the highland area followed by midland and then lowland. Overcrowding index, knowledge status of families, family member complaining of itching in the past two weeks, washing hair more than a week, wealth index, 
infrequent washing of clothes, infrequent washing of the body, history of contact with scabies patient, washing hair more than seven days and sharing of clothes were factors associated with scabies.

\section{Abbreviations}

$\mathrm{Cl}$

Confidence Interval; DALY:Disability Adjusted Life Years; EFY:Ethiopian Fiscal Year; ETB:Ethiopian Birr; GN:Glomerulonephritis; HO:Health Officer; IRB:Institute Research Board; NGOs:Non-Governmental Organizations; NTDS:Neglected Tropical Diseases; OPD:Outpatient Department; OR:Odds Ratio; PCA:Principal Component Analysis; PI:Principal Investigator; PSGN:Post streptococcal Glomerulonephritis; RF:Renal Failure; RHD:Rheumatic Heart Disease; SD:Standard Deviation; SNNPR:South Nations Nationalities Peoples Region; SPSS:Statistical Package for Social Sciences; SRS:Simple Random Sampling; UI:Uncertainty Interval; WHO:World Health Organization.

\section{Declarations}

\section{Ethical approval and consent to participate}

Ethical clearance was obtained from Arba Minch University, Institute Review Committee (IRC of AMU, Ref.No. 10994/111). Permission letters were obtained from the woreda administration office and the selected kebele leaders. The children's families were informed about the objective of the study and oral consent from each respondent's family [assent for children] from each respondent's family was obtained. The respondent's confidentiality was maintained. Those children and families who suffered from scabies and who developed secondary complications were referred to a health facility for anti-scabies medication.

Consent for publication: Not applicable

Availability of data and materials: The dataset analyzed during the study was available from the corresponding authors on reasonable request.

Competing of Interest: The authors declare that they have no competing of interest

Funding: Not applicable

\section{Authors' Contribution}

CC: Involved in generating the concept of this research paper, proposal writing, designing, analysis, write-up, preparation of the scientific paper, and read and approved manuscript $\mathrm{DH}$ : reviewed the study plan, contributed to data analysis, and read and approved manuscript. AA: reviewed the study plan, contributed to data analysis, and read and approved the manuscript. AT, BT \& EG: Involved in generating the concept of this research paper, proposal writing, designing, analysis, write-up, preparation of the scientific paper, and manuscript preparation.

\section{Acknowledgments}

We would like to thank Arba Minch University, College of Medicine and Health Sciences for their necessary support and permission to conduct the study. We are very grateful to all data collectors, supervisors, and study participants for their cooperation and assistance.

\section{References}


1. Ball, B., ed. Pediatric nursing care for children. 2 ed. 1995, Appleton and Lange publishers. 692-9.

2. CDC, Scabies-epidemiology and risk factors. [internet]. Atlanta [GA]. 2010.

3. Engelman D, K.K., Chosidow O, et al Toward the global control of human scabies: introducing the International Alliance for the Control of Scabies PLoS Negl Trop Dis, 2013. 7: p. 1-4.

4. WHO, Neglected Tropical Diseases. 2017.

5. WHO, Drought and disease outbreaks in Ethiopia: Partner update and funding request, Geneva: World Health Organization. January 2016.

6. FMOH, INTERIM-GUIDELINE FOR MULTI-SECTORIAL SCABIES OUTBREAK EMERGENCY RESPONSE ETHIOPIA. 2015.

7. A. Mahe, O.F., H. Thiam N'Diaye, F.Ly, H. Konare, S.Keita A.K. Traore, R.Hay, Definition of an algorithm for the management of common skin diseases at primary health care level in sub-Saharan Africa. Transactions of the Royal Society of Tropical Medicine and Hygiene 2005. 99: p. 39-47.

8. WHO, ed. Helminth control in school-age children: a guide for managers of control programs. 2 ed. 2011.

9. Terry BC, K.F., Sahr F et al, Sarcoptes scabies infestation among children in a displacement camp in Sierra Leone. Public Health, 2001. 115: p. 208-211.

10. Hegab DS, K.A., Kabbash IA, Dabish GM, Scabies among primary school children in Egypt: a sociomedical environmental study in Kafr El-Sheikh administrative area. Clin Cosmet Investig Dermatol, 2015. 8: p. $105-11$.

11. YOGESH G. RAUT, D.V.W., NEELAM SINGH, DR. SUSHIL AGREKAR, CROSS-SECTIONAL STUDY OF PREVALENCE OF SCABIES IN SCHOOL GOING CHILDREN. Mathematical Sciences International Research Journal, 2013. 2(2).

12. Carapetis JR, C.C., Yarmirr D, Krause V, Currie BJ, Success of a scabies control program in an Australian aboriginal community. Pediatr Infect Dis J, 1997. 16: p. 494-499.

13. Currie BJ, C.C., Krause VL, Scabies programs in aboriginal communities. Med J Aust, 1994. 161: p. 636-637.

14. Muhammad Zayyid, M., Saidatul Saadah, R., Adil, A.R., Rohela, M. and Jamaiah, I, Prevalence of scabies and head lice among children in a welfare home in Pulau Pinang, Malaysia. Tropical Biomedicine, 2010. 27(3): p. $442-446$.

15. Hosseini SJ, R.S., Noorifard M, Dabbagh-Moghaddam A, Barati M, Tabibian E, The assessment of epidemiologic aspects of scabies in Iran's Army from 2004 to 2010. Ann Mil Health Sci Res, 2014. 12(163-7).

16. Rouhullah D, M.G.A., Hamid Reza Seyyedi A, Iran Zarghi, Scabies contamination status in Iran: A review. International Journal of Epidemiologic Research, 2016. 3(1): p. 86-94.

17. Mekonnin, M., Scabies Outbreak investigations in Enarj Enawuga Woreda, East Gojjam Zone, Amhara region, Ethiopia. 2015.

18. Gulati PV, S.K., Braganza C, Role of socio-cultural and environmental factors in the cause of scabies. International journal of dermatology, 1977. 16(4): p. 281-3.

19. Hay R.J, S.A.C., Engelman. D, Walton. S Scabies in the developing world-its prevalence, complications, and management. Clin Microbiol Infect, 2012. 18: p. 313-323.

20. Nazari M. Azizi, A., Epidemiological Pattern of Scabies and Its Social Determinant Factors in West of Iran. Health, 2014. 6(1972-1977).

21. Samina Y, H.U., Muhammad Inayat Ullah K, Suleman, Sadia T et al', Epidemiological study of scabies among school-going children in district Haripur, Pakistan. Arthropods, 2017. 6(2): p. 59-66.

22. Emmanuel Armand Kouotou, J.R.N.N., Michèle K. Kouawa, Anne-Cécile Zoung-Kanyi Bissek, Prevalence and drivers of human scabies among children and adolescents living and studying in Cameroonian boarding schools.

Parasites \& Vectors, 2016. 9: p. 400.

23. Ali Hussien I, Investigation of Scabies Outbreak South- East Zone, Tigray Regional State, Ethiopia. 2016. 
24. Gurmamo, M., Scabies Outbreak Investigation, Kacha Birra District, Kembata Tembaro Zone, SNNP region, Ethiopia, November 2016.

25. Sholihah., Q., Hanafi., AS, Marilinae., L, Khairiyati., L, Fakhriadi., R et al, Relationship between Knowledge, Environmental Sanitation and Personal Hygiene with Scabies(Observational study in the Diamond Miners Community of Cempaka District Banjarbaru South Kalimantan). Scientific Research Journal (SCIRJ), 2015. III (VII).

26. Ahmed Endriss, A., SCABIES OUTBREAK INVESTIGATION IN BATI WOREDA, OROMIA ZONE AMHARA REGION NORTHWEST ETHIOPIA 2016.

27. Dr Nudrat Zeba, D.D.M.S., Dr. Khalida Naz Memon, Dr. Haji Khan Khoharo, Scabies in Relation to Hygiene and Other Factors in Patients Visiting Liaquat University Hospital, Sindh, Pakistan. International Journal of Science and Research (IJSR) August 2014. 3(8).

28. Gelaw, A., Outbreak investigation of Scabies disease, Wadela district, North wollo zone, Amhara, Ethiopia, February 2017.

29. Hermann F, A.J., Liana A, Cla'udia M, Fabı́ola A et al', The epidemiology of scabies in an impoverished community in rural Brazil: Presence and severity of disease are associated with poor living conditions and illiteracy. JAMACADDERMATOL, 2003. 60(3).

\section{Figures}

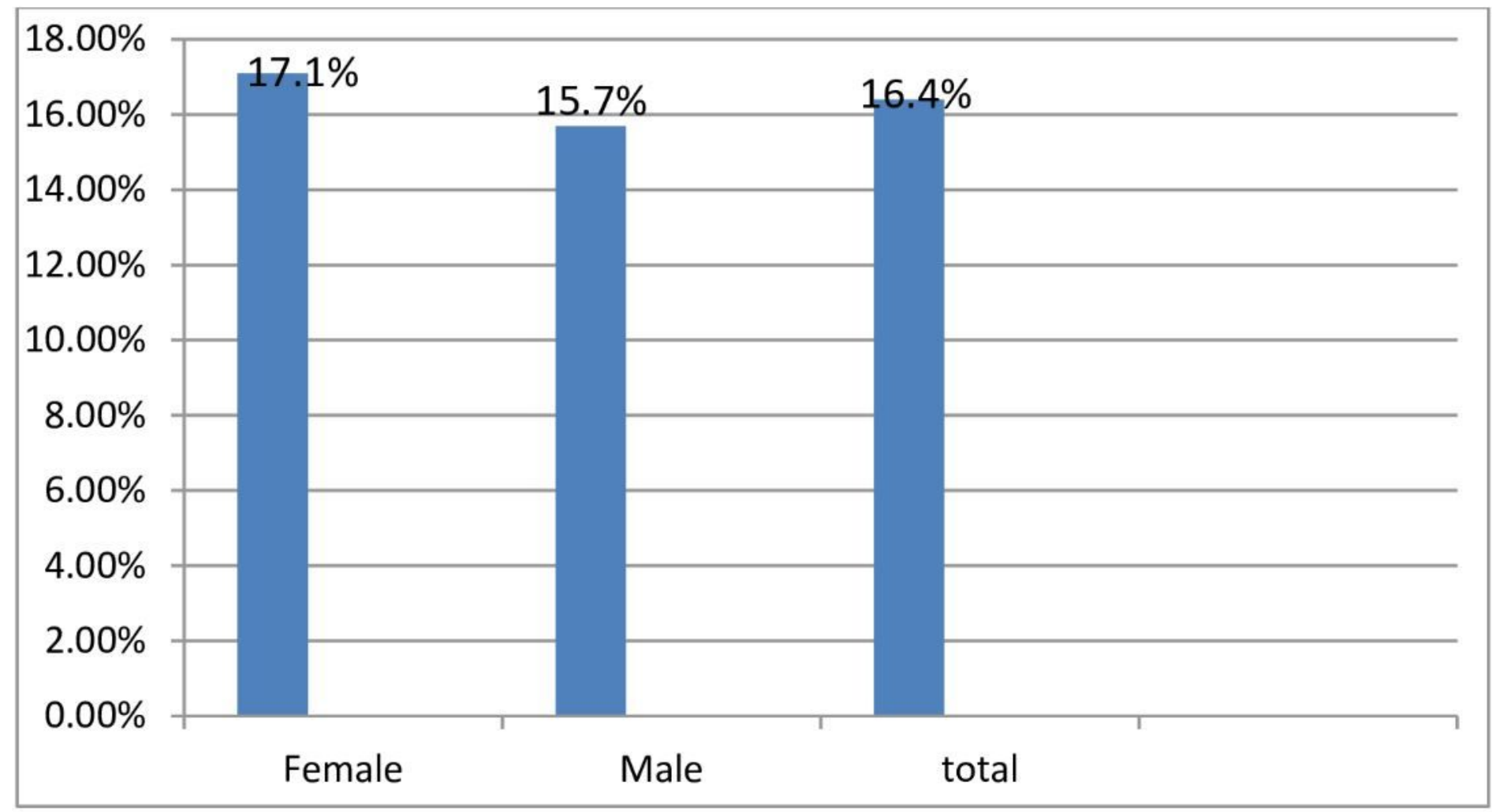

Figure 1

Distribution of prevalence of scabies by sex among school age children in Arba Munch zuria district, 2018 


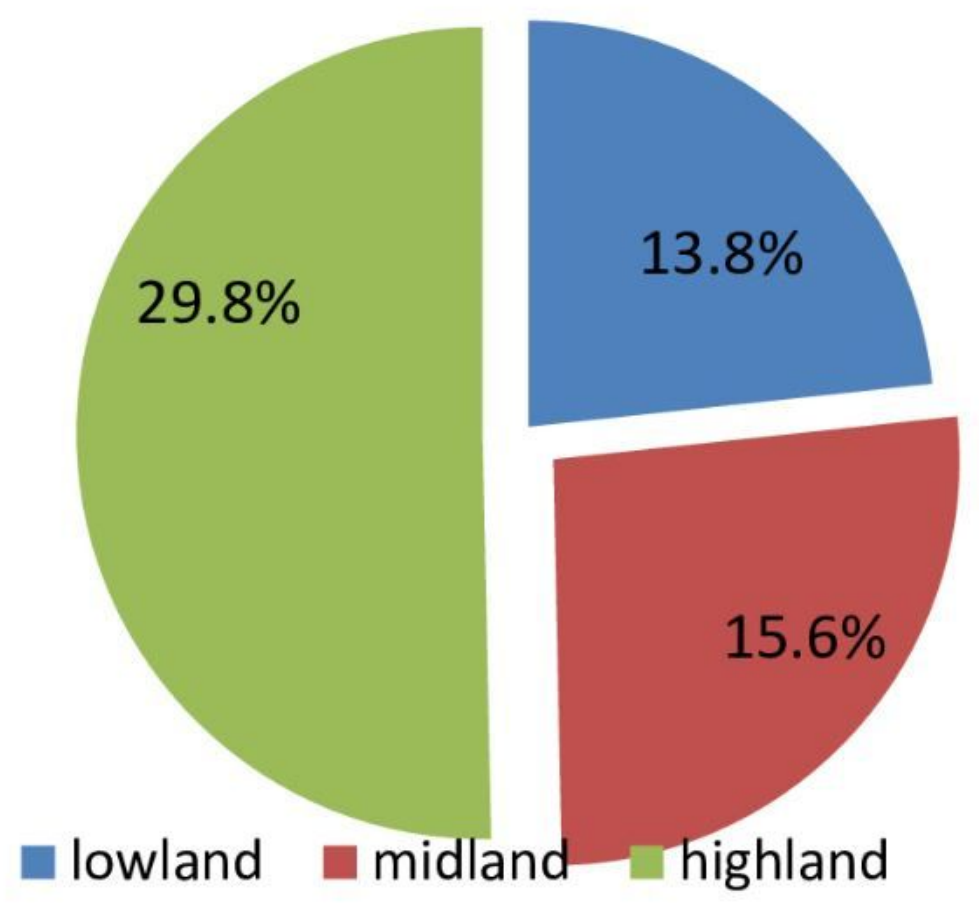

Figure 2

Distribution of scabies by climatic zone in the study area, 2018

\section{Body sites affected by scabies rash}

135

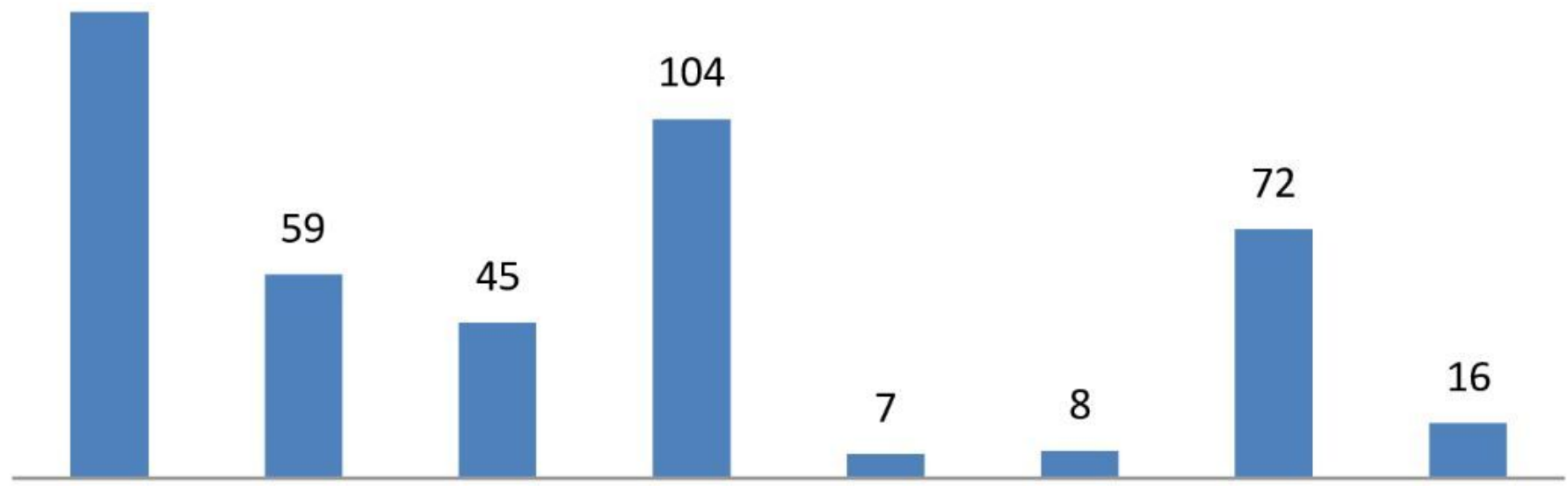

finger axulliary Genitalia abdomen shoulder Elbow Buttocks Lower webs and area blades ulnar area 


\section{Figure 3}

Objectively observed body sites affected by scabies rash among children diagnosed scabies infection in the study area, 2018; $\mathrm{N}=135$. 